\title{
Median Sternotomy After Sternal Reconstruction With Bilateral Longitudinal Plating in a Patient With Osteoporosis
}

\author{
Jeko M Madjarov, MD, ${ }^{1}$ Michael G Katz, MD, PhD,${ }^{2}$ Arvind Kumar, BS, ${ }^{2}$ Sarah M Gubara, BA, ${ }^{2}$ \\ Svetozar Madzharov, MD, ${ }^{1}$ Sophia Madjarova, BS, ${ }^{1}$ Francis Robiscek, MD, FACS, FACC ${ }^{1}$ \\ ${ }^{1}$ Department of Cardiothoracic Surgery, Sanger Heart \& Vascular Institute, Charlotte, NC, USA; ${ }^{2}$ Cardiovascular Research \\ Center, Department of Cardiology, Icahn School of Medicine at Mount Sinai, New York, NY, USA
}

\section{ABSTRACT}

In recent decades, new information has arisen regarding sternal healing and extended indications for using rigid plate fixation in patients during cardio-thoracic procedures. Three randomized controlled multicenter clinical trials recently demonstrated positive results after rigid plate fixation, including reduced sternal complications and decreased length of hospital stay. However, redo-sternotomy after sternal reconstruction utilizing rigid fixation has not been previously delineated in surgical literature. This case highlights the technical challenges of performing a median sternotomy for cardiac surgery after sternal reconstruction with bilateral longitudinal plating.

\section{INTRODUCTION}

Median sternotomy is the preferred route of access for cardiac surgery. There has been an increase in the number of patients who undergo multiple surgical procedures with median sternotomy. Wound complications resulting in instability of the sternum have contributed to the introduction of various chest closure techniques, such as simple steel wires, stainless steel coils, cables, titanium and synthetic plates, longitudinal plating etc. to enhance the stability of sternal closures in high-risk patients [Robicsek 1977; Zeitani 2006; Francel 2001; Madjarov 2018]. Three randomized, controlled, multicenter clinical trials demonstrated positive results after rigid plate fixation, including reduced sternal complications and decreased length of hospital stay [Tam 2018]. However, redo-sternotomy after chest reconstruction utilizing similar techniques of sternal closure as mentioned above has not been delineated in the surgical literature.

\section{CASE PRESENTATION}

A 69-year-old man presented to the emergency department of our institution with unstable angina. He had a history

Received October 23, 2019; accepted December 10, 2019.

Correspondence: Dr. Michael G Katz, Cardiovascular Research Center, Department of Cardiology, Icahn School of Medicine at Mount Sinai, 1470 Madison Ave, Box 1030, New York, NY 10029-6574, USA; 1-212-8248908, Fax: 1-212-241-4080 (e-mail: Michael.Katz1@mssm.edu). of ischemic heart disease and was subjected to numerous percutaneous coronary interventions and multiple stents. His other comorbidities included hypertension, chronic obstructive pulmonary disease (COPD), hypercholesterolemia, lupus erythematous treated with long-term steroids, and osteoporosis as previously confirmed by measuring bone mineral density with T-score $\leq-2.5$.

It was noted that 5 months prior, the patient was involved in a car accident and admitted to the surgical department with blunt chest trauma and transverse sternal fractures. Due to intractable, severe traumatic chest pain with compromised respiratory mechanics, the patient was offered surgical treatment and underwent uneventful sternal reconstruction with longitudinal plate fixation with prompt resolution of symptoms.

At the time of the current presentation, the patient underwent urgent coronary angiography, which revealed significant two-vessel coronary artery disease, and was then transferred to the cardiac surgery department to subsequently undergo an off-pump coronary artery bypass grafting (CABGx2). Postoperative recovery was uneventful, and the patient was discharged in stable condition on postoperative day 4 .

Technical points of a median sternotomy after previous longitudinal plate fixation

a. The technique of sternotomy must be careful and atraumatic. Extensive electro cautery to control bleeding is not well advised as it can cause tissue necrosis and a predisposition to wound complications. Careful attention to aseptic

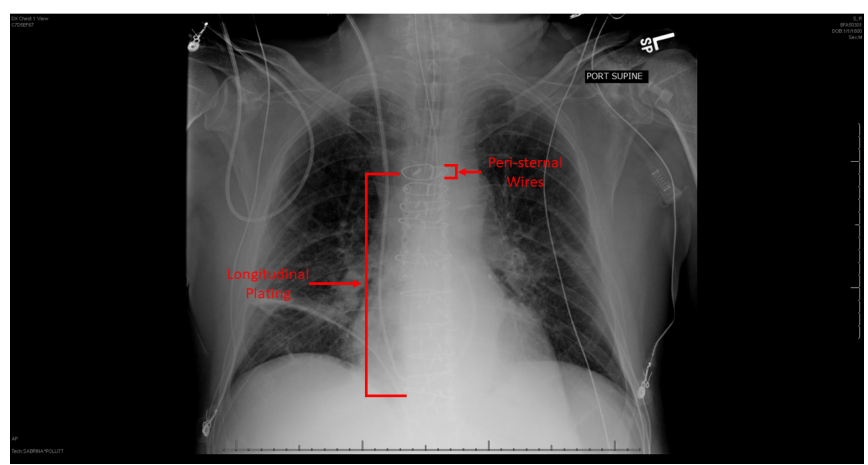

Figure 1. Postoperative chest X-Ray demonstrated peri-sternal steel wires and longitudinal plating. 


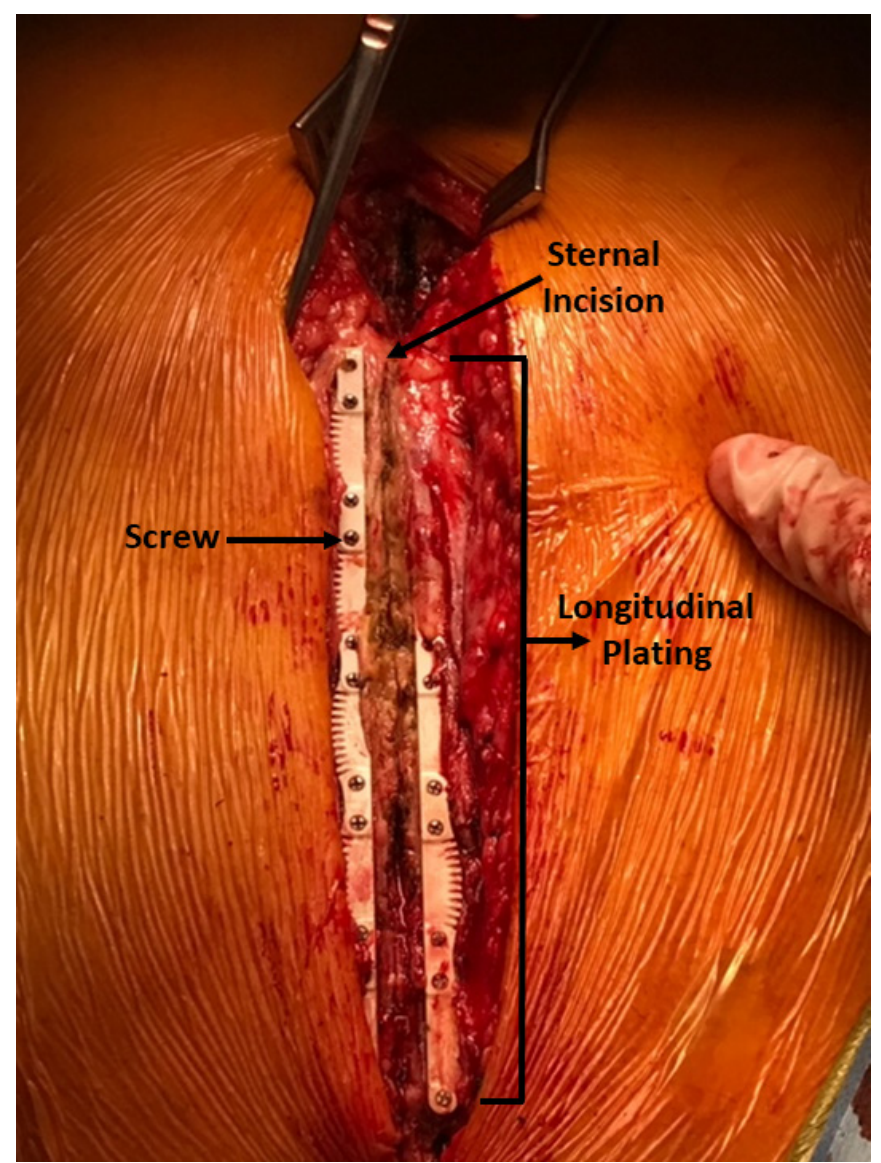

Figure 2. Intraoperative view before median sternotomy.

technique and avoidance of dead space and potential seroma formation is essential.

b. Skin and subcutaneous incision. After incision of the skin and subcutaneous layers, we dissected the pectoralis major muscle from the sternal periosteum bilaterally. In order to minimize tissue injury, minimal muscle flap dissection and elevation was performed using electro cautery at a low current setting.

c. Finding a midline. Re-sternotomy cases often lack anatomical landmarks for a midline. The midline at the sternal body can be traced precisely using objective visual cues rather than subjective palpatory feelings. The midline is conjoined to the suprasternal notch and the convergence point of the two costal arches at the linea alba. However, it also is common to discover that the traced midline is not aligned with the real midline after sternotomy.

d. Finding the plating. After finding the midline, we identified the type of plating and number of screws used in the previous procedure and correlated these findings with $\mathrm{X}$-ray images (Figure 1).

e. Sternal incision. A sternal incision with a bone saw carefully was performed between the 2 longitudinal plates, identifying any mediastinal adhesions that could cause additional bleeding. After checking the sternum for bleeding, we decided not to use bone wax and simply covered the sternal halves with towels instead. g. Harvesting of the internal mammary artery (IMA). While harvesting the IMA, we made sure to only lift the sternum enough to access the IMA. Chest retractor blades and mammary artery retractors can damage sternal halves during insertion and spreading and can cause transverse fragmentation or longitudinal separation of the sternum, especially in the settings of recent traumatic sternal fracture and repair. To better support the sternum, we placed additional longitudinal plates, which facilitated less traumatic sternal manipulation.

f. Sternal closure. The standard closure of the sternum after sternotomy usually utilizes 5 or more sutures of monofilament surgical steel passed through the sternum approximately $1 \mathrm{~cm}$ away from each side. The existing longitudinal plating allowed us to use multiple peri-sternal sutures to reinforce the sternum and to prevent wire "cut through" (Figure 2).

\section{DISCUSSION}

Sternal osteoporosis is a skeletal disease characterized by a decrease in bone strength consequent to the loss of mass and deterioration of the microstructure. These factors generally lead to the potentially significant degeneration of the bone marrow matrix as well as an increased risk of fracture [Schimmer 2008].

Mechanical protection of the fragile osteoporotic sternum is very important, during cardiac surgery via sternotomy. However, it is still unclear how to protect the structural integrity of the sternum, achieve stable closure, and prevent sternal dehiscence secondary to median sternotomy, especially in the operative settings. Analysis of our patient's sternum, previously treated with longitudinal plating, showed consistent bone healing with good new bone formation and normal bone marrow structure. The upper and lower parts of sternal segments demonstrated good healing with fibrotic scar and evidence of new bone formation.

We believe that previous sternal reconstruction with bilateral longitudinal rigid plating in patients with osteoporosis increased the bone strength of the sternum and prevented sternal complications after re-sternotomy. In our case, the previous oblique traumatic sternal fracture presented additional technical challenges. Despite the evidence of adequate bone healing, using the IMA retractor and subsequently the sternal retractor was definitely better supported by the presence of the longitudinal rigid sternal plates, which were in place from the previous, recent traumatic sternal repair.

\section{CONCLUSION}

Surgical reconstruction with bilateral longitudinal plating in patients with osteoporosis increased structural integrity of the sternum. This technique in our reported case prevented chest complications after re-sternotomy and provided mechanical protection of the fragile sternum. 


\section{REFERENCES}

Francel TJ, Kouchoukos NT. 2001. A rational approach to wound difficulties after sternotomy: reconstruction and long-term results. Ann Thorac Surg 72:1419-29.

Madjarov JM, Katz MG, Kane PN, Madzharov S, Robicsek F. 2018. Early surgical reconstruction of sternum with longitudinal rigid polymer plating after acute chest trauma. Ann Thorac Cardiovasc Surg 24:324-327.

Robicsek F, Daugherty HK, Cook JW. 1977. The prevention and treatment of sternum separation following open-heart surgery. J Thorac
Cardiovasc Surg 73:267-8.

Schimmer C, Sommer SP, Bensch M, Bohrer T, Aleksic I, et al. 2008. Sternal closure techniques and postoperative sternal wound complications in elderly patients. Eur J Cardiothorac Surg 34:132-8.

Tam DY, Nedadur R, Yu M, Yanagawa B, Fremes SE, et al. 2018. Rigid plate fixation versus wire cerclage for sternotomy after cardiac surgery: a meta-analysis. Ann Thorac Surg 106:298-304.

Zeitani J, Penta de Peppo A, Moscarelli M, et al. 2006. Influence of sternal size and inadvertent paramedian sternotomy on stability of the closure site: a clinical and mechanical study. J Thorac Cardiovasc Surg $132: 38-42$ 